\title{
The PRY/SPRY Domain of Pyrin/TRIM20, The Familial Mediterranean Fever Protein, Interacts with B2-Microglobulin to Promote Inflammasome Formation
}

\section{Sei Samukawa}

Yokohama City University School of Medicine Graduate School of Medicine: Yokohama Shiritsu Daigaku Igakubu Daigakuin Igaku Kenkyuka

Ryusuke Yoshimi ( $\nabla$ yoshiryu@med.yokohama-cu.ac.jp)

Yokohama City University School of Medicine https://orcid.org/0000-0002-3945-307X

\section{Yohei Kirino}

Yokohama City University School of Medicine Graduate School of Medicine: Yokohama Shiritsu Daigaku Igakubu Daigakuin Igaku Kenkyuka

\section{Hideaki Nakajima}

Yokohama City University School of Medicine Graduate School of Medicine: Yokohama Shiritsu Daigaku Igakubu Daigakuin Igaku Kenkyuka

\section{Research Article}

Keywords: Pyrin, TRIM family, inflammasome, $\beta 2$-microglobulin, familial Mediterranean fever

Posted Date: February 19th, 2021

DOl: https://doi.org/10.21203/rs.3.rs-212639/v1

License: (c) (i) This work is licensed under a Creative Commons Attribution 4.0 International License. Read Full License 


\section{Abstract}

Background. Pyrin/TRIM20 is expressed in the neutrophils and monocytes/macrophages and regulates caspase- 1 activation and interleukin-1 $\beta$ maturation. Although the mutations in the PRY/SPRY domain of pyrin cause familial Mediterranean fever (FMF), the mechanism of how mutated pyrin provokes excessive inflammation in FMF patients is not well understood. This study was undertaken to explore the new pyrin $\mathrm{PRY} / \mathrm{SPRY}$ domain-binding protein and to investigate how the interaction affected the pyrin function.

Methods. We carried out the yeast two-hybrid screening for pyrin PRY/SPRY domain-binding proteins. Then, protein interactions were investigated using Lenti-X 293T cells expressing pyrin-associated proteins by co-immunoprecipitation analysis. We also used human embryonic kidney (HEK) 293 cells expressing pyrin-associated proteins and human neutrophils stimulated with monosodium urate crystal for immunofluorescence staining analysis.

Results. $\beta_{2}$-Microglobulin ( $\beta 2 M G$ ) was identified as the novel pyrin ligand binding to the PRY/SPRY domain. $\beta 2 M G$ was co-localized with pyrin not only in the HEK293 cells overexpressing these proteins but also in the stimulated human neutrophils in the speck-like structures. The pyrin- $\beta 2 M G$ interaction triggered the binding of pyrin and proline-serine-threonine phosphatase interacting protein 1 (PSTPIP1) and then the subsequent recruitment of apoptosis-associated speck-like protein containing caspase recruitment domain (ASC). Caspase-1 p20 subunit, produced by pyrin inflammasome, also interacted with the pyrin PRY/SPRY domain and inhibited the pyrin- $32 M G$ interaction. FMF-associated pyrin mutation M694V did not affect pyrin- $\beta 2 M G$ interaction but weakened this inhibition.

Conclusions. Our findings suggest that $\beta 2 M G$ functions as the pyrin ligand inducing pyrin inflammasome formation and that the FMF-associated pyrin mutations weakened negative feedback of caspase-1 p20 subunit.

\section{Background}

Familial Mediterranean fever (FMF) is one of the most prevalent hereditary autoinflammatory diseases, characterized by recurrent febrile attacks with serosal inflammation (1). The onset of FMF is usually in childhood and the patients show periodical episodes of fever with chest and/or abdominal pain, which resolve within 48-72 hours. The gene responsible for FMF was identified and named MEFV, which is located on chromosome 16, in $1997(2,3)$.

The MEFV gene encodes pyrin protein, which is also known as tripartite motif-containing (TRIM) 20 or marenostrin. Pyrin is a 781-amino acid and $\sim 86 \mathrm{kDa}$ protein, which is mainly expressed in the cytoplasm of the cells of the innate immune system, including neutrophils, eosinophils, monocytes, macrophages, and dendritic cells (4). Pyrin belongs to the TRIM family, which numbers over 70 proteins in humans with homologs identified in many species ranging from primates to nematodes, exhibiting a wide range of 
activities, including the regulation of innate and adaptive immune responses (5-9). Pyrin is comprised of several distinct domains- pyrin, B-box and coiled-coil domain in the $\mathrm{N}$-terminal region followed by a PRY/SPRY domain in the C-terminus (10).

Pyrin interacts with an adapter protein called apoptosis-associated speck-like protein with a caspaserecruitment domain (ASC) through the interaction of their respective $\mathrm{N}$-terminal pyrin domains (11). ASC oligomerizes and mediates the proteolytic activation of caspase-1, leading to proteolytic maturation and secretion of interleukin-1 $\beta$ (IL-1 $\beta$ ) in cytosolic multiprotein oligomers denoted inflammasomes (12). Therefore, it is presumed that pyrin has a proinflammatory role by forming "pyrin inflammasome" with ASC and caspase-1 (13).

So far, several pathways have been suggested to induce the pyrin inflammasome formation. One of them is proline-serine-threonine phosphatase-interacting protein 1 (PSTPIP1)-mediated pathway. PSTPIP1 binds to the B-box domain of pyrin (14), and its mutation causes another hereditary autoinflammatory disease, pyogenic arthritis, pyoderma gangrenosum and acne (PAPA) syndrome (15). PAPA syndromeassociated PSTPIP1 mutation, A230T, heightens its affinity for pyrin and consequently promotes the pyrin inflammasome formation (14). However, it remains unknown what triggers the interaction between wildtype PSTPIP1 and pyrin in FMF pathology.

Because FMF-associated MEFV mutations are concentrated on exon 10 (16), corresponding to PRY/SPRY domain of pyrin (e.g. M680I, M694I/V, and V726A), this domain has been considered essential for the molecular mechanisms causing FMF. The crystal structure of the pyrin PRY/SPRY domain forms a shallow cavity, which seems to be a protein binding site, and FMF-associated mutation sites are observed around it (17), suggesting that FMF-associated pyrin mutations affect the binding affinity of its ligands. To date, the p20 subunit of caspase-1 (18) and apoptosis-associated protein Siva-2 (19) were reported to bind to the pyrin PRY/SPRY domain.

There are two conflicting studies investigating the effect of FMF-associated pyrin mutation on the binding affinity of PRY/SPRY domain to p20. A study group reported that pyrin-p20 binding was reduced by FMF-associated mutations in PRY/SPRY domain (18). They also showed that FMF-associated mutations and deletion of the PRY/SPRY domain reduce the effect of pyrin on the attenuation of IL-1 $\beta$ production. On the other hand, the other group reported that pyrin M694V mutation, the most frequent mutation found in FMF patients, showed no impacts on the interaction of pyrin with p20 (20). The result suggests that the FMF-associated mutations do not affect the pyrin-p20 interaction directly but indirectly by some other pyrin-binding proteins whose affinity for pyrin is affected by the mutations.

Although the previous studies shed some light on pyrin and FMF pathogenesis as just described, it is still unclear how the FMF-associated pyrin mutations lead to inflammasome activation and IL-1 $\beta$ overproduction. In the present work, we demonstrate that $\beta_{2}$-microglobulin ( $\beta 2 \mathrm{MG}$ ) associates and colocalizes with pyrin in the cytoplasm. $\beta 2 \mathrm{MG}$ interacts with PRY/SPRY domain of pyrin, in more detail, with the hotspot of FMF-associated mutations, and promotes PSTPIP1-mediated ASC recruitment. The 
$32 \mathrm{MG}$-pyrin association is suppressed by the $\mathrm{p} 20$ subunit of caspase-1. Pyrin M694V mutation promotes the $32 \mathrm{MG}$-pyrin association in the presence of $\mathrm{p} 20$. The mutation itself does not affect pyrin-PSTPIP1 interaction in the presence of $\beta 2 \mathrm{MG}$. Taken together, our results point to the importance of $\beta 2 \mathrm{MG}$-pyrin interaction in the molecular mechanism of pyrin inflammasome activation in FMF.

\section{Methods}

\section{Yeast two-hybrid library screening}

Yeast two-hybrid screening was performed using the Matchmaker Gold Two-hybrid System (Clontech Laboratories, Palo Alto, CA). To construct bait plasmids, full-length human pyrin (FL-pyrin) and the Cterminal region of human pyrin (C-pyrin; aa 598-781) was cloned in frame with the GAL4 DNA-binding domain of pGBKT7 vector. The yeast strain AH109 was transformed with pGBKT7-FL-pyrin and pGBKT7C-pyrin, respectively. As prey plasmids, human leukocyte cDNA library was inserted downstream of the GAL4 activation domain of pACT2 vector (Clontech Laboratories), and then the yeast strain Y187 was transformed with the plasmids. After mating the $\mathrm{AH} 109$ and $\mathrm{Y} 187$ transformants, they were spread on plates with high selectivity medium (synthetic drop-out medium without adenine, histidine, tryptophan, and leucine). After identifying the positive colonies by $\beta$-galactosidase activity, cDNAs inserted in the prey vector were amplified by colony-direct PCR. The positive inserts were sequenced and analyzed using the BLAST program (National Center for Biotechnology Information, Bethesda, MD).

\section{Antibodies (Abs)}

Anti-FLAG monoclonal and polyclonal Abs were obtained from Sigma-Aldrich. Anti-V5 monoclonal Ab was obtained from Invitrogen. Anti-pyrin rabbit polyclonal Ab and anti- $\beta 2 M G$ mouse monoclonal Ab were from Sigma-Aldrich.

\section{Monosodium urate (MSU) crystal preparation}

MSU crystals were prepared by the method described by Seegmiller et al. (21). Briefly, uric acid (SigmaAldrich) was dissolved in boiling water, and the $\mathrm{pH}$ of the solution was adjusted to 7.2 by adding $\mathrm{NaOH}$. Then the solution was cooled slowly while stirring at room temperature until MSU crystals precipitated. The crystals were washed with ethanol, autoclaved, suspended in PBS at a concentration of $10 \mathrm{mg} / \mathrm{ml}$, and then sonicated to obtain rod-shaped crystals.

\section{Cell culture}

Human embryonic kidney (HEK) 293 cells and Lenti-X 293T (Clontech Laboratories) were cultured at $37^{\circ} \mathrm{C}$ in a humidified atmosphere of $5 \% \mathrm{CO} 2$ in DMEM medium containing $10 \% \mathrm{FBS}, 100 \mathrm{U} / \mathrm{ml}$ penicillin, and $100 \mu \mathrm{g} / \mathrm{ml}$ streptomycin.

Human neutrophils were isolated from the peripheral blood of healthy donors by centrifugation using Polymorphprep (Abbot diagnostic technologies AS) as a manufacturer's instruction. Purified neutrophils were resuspended in RPMI medium with 10\% FBS and cultured in 35-mm dishes at the concentration of 2 
$\times 10^{5}$ cells per dish. Neutrophils were primed with $500 \mathrm{ng} / \mathrm{ml}$ Salmonella enteritica lipopolysaccharide (LPS; Sigma-Aldrich) for $3 \mathrm{~h}$ and then stimulated with $200 \mu \mathrm{g} / \mathrm{ml}$ MSU crystals for $2 \mathrm{~h}$, which were subjected to immunofluorescence analyses.

\section{Transfection}

For HEK293 cells, plasmid transfection was performed by lipofection using lipofectamine LTX (Invitrogen) for immunofluorescence analyses. For Lenti-X 293T cells, plasmid transfection was performed using calcium phosphate method and for immunoprecipitation assays and Western blotting.

\section{Immunoprecipitation assay}

Lenti-X 293T cells $\left(2.0-4.0 \times 10^{6}\right.$ cells) transfected with the expression plasmids (see Supplemental Table 1 for usage) were lysed with the $500 \mu$ l of lysis buffer ( $20 \mathrm{mM}$ Tris- $\mathrm{HCl}(\mathrm{pH} \mathrm{6.0)}, 120 \mathrm{mM} \mathrm{NaCl}, 5 \%$ glycerol, and $0.5 \%$ Triton X-100). After clarification by centrifugation at $20,000 \times g$ for 30 min, each $20 \mu \mathrm{l}$ of these lysates was used as supernatant, and the remaining were incubated with $5 \mu$ l of agarose beads conjugated with anti-FLAG Ab (Sigma-Aldrich) or $10 \mu$ lof magnet beads conjugated with anti-V5 Ab (Medical \& Biological Laboratories) for $1 \mathrm{~h}$ at $4^{\circ} \mathrm{C}$. After extensive washing with the lysis buffer, the immunocomplexes were solubilized by adding SDS sample buffer to the beads and subjected to Western blot analysis using a chemiluminescence ECL system (Amersham Biosciences). Anti-FLAG and anti-V5 Abs were diluted to 1:5,000 for Western blot analyses. $\beta$-Actin was detected as a loading control using 1:10,000 dilution of Direct-Blot HRP anti- $\beta$-actin Antibody (BioLegend). We performed each experiment at least three times to confirm reproducibility. Each data in the figures show a representative of them.

\section{Immunofluorescence staining}

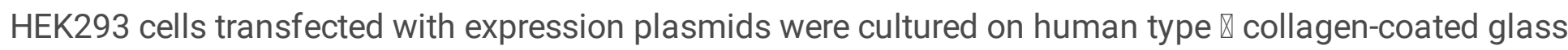
chamber slides (BD biosciences). After washed with PBS, they were fixed with $2 \%$ paraformaldehyde in PBS for $15 \mathrm{~min}$ at room temperature and then permeabilized with PBS containing $0.5 \%$ Triton X-100 for 10 min. After blocking with PBS containing $10 \%$ BSA, the cells were treated with appropriate primary Abs (rabbit anti-FLAG polyclonal $\mathrm{Ab}$ and mouse anti-V5 monoclonal $\mathrm{Ab}$ ) for $1 \mathrm{~h}$ at $37^{\circ} \mathrm{C}$ in a moist chamber, washed with PBS containing $0.05 \%$ Tween 20, and incubated with secondary Abs (Alexa Flour 488 donkey anti-mouse IgG and Alexa Fluor 555 goat anti-rabbit IgG Abs (Amersham Biosciences)). After washing, the samples were observed under a fluorescence microscope, BZ9000 (Keyence).

Neutrophils with or without stimulation were collected by centrifugation at $500 \times \mathrm{g}$ for $10 \mathrm{~min}$. After washed with PBS, fixed, permeabilized, and blocked as described above, the cells were treated with primary Abs (rabbit anti-pyrin polyclonal $A b$ and mouse anti- $\beta 2 M G A b$ ) diluted to 1:1,000. After washing, the cells were incubated with the secondary Abs. We performed each experiment at least three times to confirm reproducibility. Each data in the figures show a representative of them.

\section{Results}


Yeast two-hybrid screening for pyrin-binding protein.

Although certain bacterial toxins induce pyrin inflammasome formation (22), FMF attacks are not always triggered by infection but some other factor including stress and menstrual cycle. Therefore, we hypothesized that some endogenous proteins expressed in neutrophil may interact with pyrin and play an important role as a damage-associated molecular pattern. To identify a molecule that interacts with pyrin, we performed a yeast two-hybrid screening assay using human leukocyte cDNA library as prey. Our use of full-length pyrin (FL-pyrin; aa 1-781) as bait yielded 60 positive colonies (Fig. 1). To exclude false positives as much as possible before subsequent confirmatory binding assays, and to identify pyrinbinding proteins which interact with pyrin via its PRY/SPRY (B30.2) domain where mutations are often observed in patients with FMF, we also performed the screening using the C-terminal region of pyrin molecule (C-pyrin; aa 598-781) as bait. We found 124 positive colonies among which only 9 were isolated from both screenings using FL-pyrin bait and that using C-pyrin bait in common (Fig. 1). The colony-direct PCR and sequence revealed the 9 candidates for genes encoding pyrin-binding molecules, including FTH1, EEF1A1, TPT1, COX5A, CYBA, B2M, ACTB, FLOT1, and LTB (Table 1).

Table 1

The genes obtained commonly from both yeast two-hybrid library screenings using FL-and C-pyrin baits.

\begin{tabular}{|c|c|c|}
\hline $\begin{array}{l}\text { Symbol/Gene } \\
\text { ID }\end{array}$ & Full Name & Summary \\
\hline FTH1/2945 & ferritin, heavy polypeptide 1 & Iron storage protein \\
\hline EEF1A1/1915 & $\begin{array}{l}\text { eukaryotic translation elongation } \\
\text { factor } 1 \text { alpha } 1\end{array}$ & $\begin{array}{l}\text { Enzymatic delivery of aminoacyl tRNAs to the } \\
\text { ribosome }\end{array}$ \\
\hline TPT1/7178 & $\begin{array}{l}\text { tumor protein, translationally- } \\
\text { controlled } 1\end{array}$ & Regulator of cellular growth and proliferation \\
\hline COX5A/9377 & $\begin{array}{l}\text { cytochrome c oxidase subunit } \\
\text { Va }\end{array}$ & $\begin{array}{l}\text { Terminal enzyme of the mitochondrial } \\
\text { respiratory chain }\end{array}$ \\
\hline CYBA/1535 & $\begin{array}{l}\text { cytochrome b-245, alpha } \\
\text { polypeptide }\end{array}$ & $\begin{array}{l}\text { Primary component of the microbicidal oxidase } \\
\text { system of phagocytes }\end{array}$ \\
\hline$B 2 M / 567$ & beta-2 microglobulin & 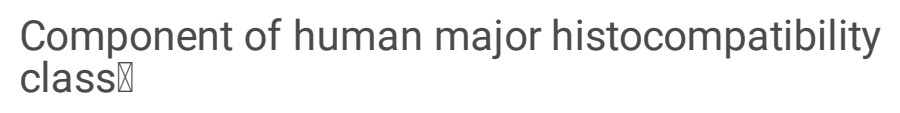 \\
\hline ACTB/60 & actin beta & Cytoskeleton \\
\hline FLOT1/10211 & flotillin 1 & Vesicle trafficking and cell morphology \\
\hline LTB/4050 & lymphotoxin beta & Inducer of the inflammatory response system \\
\hline
\end{tabular}

$\beta 2 \mathrm{MG}$ interacts with pyrin.

32MG protein is a component of major histocompatibility complex $(\mathrm{MHC})$ class I molecules, which are present on the plasma membrane of all nucleated cells. In neutrophils, $\beta 2 M G$ molecules are expressed not only on the cell surface as an MHC class I component but also inside the cells (23). Two-thirds of 
$32 \mathrm{MG}$ expression amount in neutrophils is localized in gelatinase granules or specific granules although the physiological role of this intragranular $\beta 2 M G$ is still unknown (23). As we identified the $B 2 M$ gene, which encodes $\beta 2 \mathrm{MG}$ protein, as the candidate for genes encoding pyrin-binding molecules by the yeast two-hybrid screening, we focused on $\beta 2 M G$ and tried to confirm the interaction with pyrin by immunoprecipitation assay. We subcloned the full-length B2M cDNA into pcDNA3.1(-) vectors with V5 tag in the N-terminus and co-transfected them with FLAG-tagged pyrin expression vector into Lenti-X 293T cells. The co-immunoprecipitation analysis using anti-FLAG Ab revealed that $\mathrm{V} 5$-tagged $\beta 2 \mathrm{MG}$ was coprecipitated with FLAG-tagged pyrin (Fig. 2A). Additionally, the reciprocal immunoprecipitation assay using anti-V5 Ab showed that FLAG-pyrin was co-precipitated with V5-32MG (Fig. 2B). To further confirm the pyrin- $\beta 2 M G$ interaction, the expression vectors for FLAG-pyrin and V5- $\beta 2 M G$ were co-transfected into HEK293 cells and the localization of these fusion proteins was examined by immunofluorescence staining. As shown in Fig. $2 \mathrm{C}$, the overexpressed V5- $\beta 2 \mathrm{MG}$ distributed homogeneously in the cytoplasm and did not show any specific structure when FLAG-pyrin was not overexpressed. On the other hand, V532MG co-localized with pyrin in the speck-like structures in the perinuclear position in more than $90 \%$ of the cells in which both FLAG-pyrin and V5- $\beta 2 M G$ were overexpressed.

To confirm the localization of endogenous pyrin and $\beta 2 M G$, we examined human neutrophils isolated from the peripheral blood of healthy donors and stained with anti-pyrin and anti- $\beta 2 M G$ Abs. $\beta 2 M G$ staining was not co-localized with pyrin staining in neutrophils without any stimulation (Fig. 3, upper pane/s). Because interaction between neutrophils and MSU crystals has been shown to elicit various responses including IL-1 $\beta$ secretion (24), we next investigated their localization in the stimulated neutrophils. Interestingly, $\beta 2 M G$ was co-localized with pyrin in neutrophils stimulated with LPS and MSU in the speck-like structures (Fig. 3, lower pane/s), suggesting that the pyrin- $\beta 2 \mathrm{MG}$ interaction is induced by neutrophil activation.

$\beta 2 M G$ binds to the hotspot of FMF-associated mutations of pyrin.

Because the B2M gene was identified not only by yeast two-hybrid screening using FL-pyrin bait but also using C-pyrin bait, we expected that $\beta 2 M G$ can bind to the PRY/SPRY domain of pyrin. To further investigate the binding site for $\beta 2 M G$ in pyrin molecule, we constructed the vectors which express FLAGtagged pyrin lacking PRY/SPRY domain corresponding to exon 10 ( $\triangle \mathrm{E} 10$ pyrin) and pyrin PRY/SPRY domain (E10 pyrin) and transfected them with V5- $\beta 2 M G$ expression vector into Lenti-X 293T cells. Although FLAG-E10 pyrin co-precipitated V5-32MG, FLAG- $\triangle$ E10 pyrin did not (Fig. 4A). The reciprocal immunoprecipitation assay using anti-V5 Ab showed that FLAG-E10 pyrin was co-precipitated with V5B2MG (Fig. 4B). Consistent with the data, immunofluorescence staining revealed that the overexpressed V5- $\beta 2 M G$ was not colocalized with FLAG- $\triangle$ E10 pyrin but with FLAG-E10 in speck-like structures (Fig. 4C). These results indicate that pyrin interacts with $\beta 2 M G$ via its PRY/SPRY domain.

FMF-associated mutations accumulate in the middle portion of the pyrin PRY/SPRY domain (Fig. 5A). Among them, M680I, M694V, M694I, and V726A are listed as the minimum set of clearly pathogenic sequence variants recommended to screen in the genetic diagnostic guidelines developed by Shinar et al. 
(25). To elucidate the relationship between these mutations and $\beta 2 \mathrm{MG}$ binding, we constructed expression vectors for FLAG-tagged pyrin deletion mutants, pyrin E10-1 (aa 598-659), pyrin E10-2 (aa 660-727) and pyrin E10-3 (aa 728-781). These expression vectors were co-transfected with V5-tagged B2MG into Lenti-X 293T cells and subjected to co-immunoprecipitation analysis. As shown in Fig. 5B, all FLAG-tagged E10 pyrin mutants could co-precipitate V5-32MG. However, FLAG-tagged pyrin E10-2, which includes the hotspot of FMF-associated mutations, co-precipitated V5- $\beta 2 M G$ much more strongly as compared to other E10 mutants, suggesting the important role of mutations in the pyrin- $\beta 2 \mathrm{MG}$ interaction.

32MG-pyrin interaction recruits PSTPIP1 leading to the assembly of pyrin inflammasome.

PSTPIP1, which was identified as a genetic cause of another autoinflammatory disorder, PAPA syndrome, has been shown to interact with the B-box/coiled-coil domain of pyrin (26). Furthermore, PSTPIP binding has been shown to activate pyrin by unmasking the pyrin domain, promoting ASC-mediated oligomerization and inflammasome formation (14). Thus, we next investigated whether pyrin- $32 \mathrm{MG}$ interaction affects pyrin-ASC and pyrin-PSTPIP1 interactions. As shown in Fig. 6A, when HEK293 cells overexpressing FLAG-tagged pyrin and V5-tagged ASC were subjected to immunofluorescence staining, FLAG-pyrin and V5-ASC were co-localized in the cytoplasm with the speck formation (arrows in the light field images), indicating pyrin-ASC interaction. To clarify the effect of pyrin- $\beta 2 \mathrm{MG}$ interaction on pyrinASC interaction, FLAG-tagged pyrin, and V5-tagged ASC expression vectors were transfected into Lenti-X 293T cells with or without V5-tagged $\beta 2 M G$ expression vector. The co-immunoprecipitation analysis showed that pyrin did not interact with ASC (Fig. 6B) and $32 \mathrm{MG}$ overexpression did not induce pyrin-ASC interaction (Fig. 6C).

We next examined the effect of pyrin- $32 \mathrm{MG}$ interaction on pyrin-PSTPIP1 interaction. First, we investigated the interaction between pyrin, PSTPIP1, and ASC in the absence of $\beta 2 M G$ overexpression by the co-immunoprecipitation analysis using FLAG-pyrin, V5-ASC expression vectors together with V5tagged wild-type PSTPIP1 or one of the most common PAPA-associated PSTPIP1 mutation, A230T. FLAG-pyrin co-precipitated A230T PSTPIP1 drastically as compared to the wild-type PSTPIP1 (Fig. 6D), consistent with the previous study showing that A230T mutation induces its hyperphosphorylation and increases the affinity to pyrin (26). Additionally, the pyrin-ASC interaction was also promoted by the mutation, indicating that the PSTPIP1 mutation increases the pyrin inflammasome formation (Fig. 6D). Then, FLAG-pyrin and V5-PSTPIP1 expression vectors were transfected into Lenti-X 293T cells with or without a V5- $\beta 2 M G$ expression vector. Co-immunoprecipitation analysis revealed that $\beta 2 M G$ overexpression drastically promoted the pyrin-PSTPIP1 interaction (Fig. 6E).

To assess whether $\beta 2 M G$ promotes ASC recruitment, we have overexpressed V5- $\beta 2 M G$ together with pyrin, PSTPIP1, and ASC in the cells, and investigated the change of ASC recruitment by the $\beta 2 \mathrm{MG}$ mediated enhancement of pyrin-PSTPIP1 interaction. As a result, $\beta 2 \mathrm{MG}$ overexpression promoted ASC recruitment (Fig. 6F). The ability is PSTPIP1-dependent because the induction of ASC recruitment was not 
observed without PSTPIP1 overexpression (Fig. $6 \mathrm{C}$ ). These results suggest that $\beta 2 \mathrm{MG}$ functions as a pyrin-binding protein inducing PSTPIP1-mediated pyrin inflammasome formation.

Caspase-1 p20 subunit inhibits the association of $\beta 2 M G$ with pyrin.

The activation of caspase- 1 is caused by the autocleavage of pro-caspase- 1 , which leads to the formation of active caspase- 1 p10/p20 tetramer (27). It is a pivotal process for IL-1 $\beta$ and IL-18 production after the assembly of the pyrin inflammasome. On the other hand, p20 and p10 have been shown to interact with the PRY/SPRY domain of pyrin directly, resulting in the inhibitory effect on IL-1 $\beta$ production (18). Thus, we hypothesized that the binding of $\beta 2 M G$ and caspase- 1 to pyrin may compete on the pyrin PRY/SPRY domain. We constructed the expression vectors for V5-tagged caspase-1 p20 and transfected them into the Lenti-X 293T cells with the expression vectors for FLAG-tagged $\Delta \mathrm{E} 10$ or E10 pyrin. Coimmunoprecipitation analysis revealed that caspase- 1 p20 interacts with the PRY/SPRY domain of pyrin as shown in the previous report (Fig. 7A) (18). To investigate the relationship between the FMFassociated pyrin mutations and caspase- 1 binding, we co-transfected the vectors for FLAG-tagged pyrin deletion mutants, pyrin E10-1, E10-2 or E10-3 with V5-tagged p20 into Lenti-X 293T cells, and subjected them to co-immunoprecipitation analysis. As is the case with $\beta 2 M G$, FLAG-tagged E10-2, which includes the hotspot of FMF-associated mutations, co-precipitated V5-p20 best among the pyrin deletion mutants (Fig. 7B). To elucidate whether caspase-1 has an inhibitory effect on pyrin- $\beta 2 M G$ interaction, FLAGtagged pyrin and V5-tagged $\beta 2 \mathrm{MG}$ expression vectors were co-transfected into Lenti-X 293T cells with or without V5-tagged caspase-1 p20 expression vector. Co-immunoprecipitation analysis revealed that overexpression of caspase-1 p20 inhibits the interaction between pyrin and $32 \mathrm{MG}$ (Fig. 7C). These results suggest that p20 acts as an inhibitor for $\beta 2 M G$-mediated promotion of pyrin inflammasome formation and suppresses the excess inflammation via a negative feedback mechanism.

Pyrin M694V mutation changes the balance of affinities toß2MG and caspase-1 p20.

Homozygous M694V mutation can cause severer forms of FMF and AA amyloidosis in higher prevalence (28). To elucidate whether M694V mutation affects $\beta 2 \mathrm{MG}$ affinity for pyrin, we constructed expression vectors for FLAG-tagged full-length pyrin and E10-2 fragment with M694V mutation and compared the B2MG affinity between for M694V-mutated pyrin or E10-2 fragment and for the wild type by coimmunoprecipitation assay. As shown in Fig. 8A and 8B, the M694V mutation did not make any impact on the $\beta 2 M G$ affinity in the absence of caspase-1 p20 overexpression. Because our data showed that caspase-1 p20 inhibits the association of $\beta 2 \mathrm{MG}$ with pyrin, we next investigated the effect of M694V mutation on inhibition of pyrin- $32 \mathrm{MG}$ interaction by $\mathrm{p} 20$. Unlike wild-type pyrin, M694V-mutated pyrin coprecipitated $\beta 2 M G$ even in the presence of p20, suggesting that pyrin M694V mutation weakened negative feedback on PSTPIP1-mediated pyrin inflammasome formation by caspase-1 p20 (Fig. 8C).

Finally, we examined whether pyrin M694V mutation affects PSTPIP1 recruitment by coimmunoprecipitation analysis. As a result, M694V-mutation itself did not affect the pyrin-PSTPIP1 interaction in the presence of $\beta 2 \mathrm{MG}$ (Fig. 8D). These findings suggest that pyrin M694V mutation leads to 
the overproduction of pyrin inflammasome not by promoting $32 \mathrm{MG}$-induced PSTPIP1 recruitment but by reduction of the inhibitory effect of caspase-1 p20 on pyrin- $\beta 2 M G$ interaction.

\section{Discussion}

Although the function of pyrin protein, encoded by the MEFV gene, has been investigated by many researchers since MEFV was identified as the responsible gene for FMF, it has not been completely understood how MEFV mutations affect the function of pyrin. In this study, we identified $\beta 2 M G$ as a protein that binds to the pyrin PRY/SPRY domain, which was competitively blocked by caspase-1 p20 subunit. $\beta 2 M G$-pyrin interaction leads to the recruitment of PSTPIP1, a crucial component of pyrin inflammasome. Pyrin M694V mutation strengthened the $\beta 2 M G-p y r i n$ interaction.

Recently, several papers have shown that RhoA GTPase indirectly regulates pyrin inflammasome activation. RhoA-dependent serine/threonine protein kinases PKN1 and PKN2 phosphorylate pyrin at S208 and S242 residues, which are located between pyrin and B-box domains, and 14-3-3 protein traps the phosphorylated pyrin, preventing pyrin from inflammasome formation (29). They also elucidated that some bacterial toxins, such as TcdB from Clostridium difficile, inhibit the activity of RhoA, leading to a decrease of PKN1/2 activities and pyrin phosphorylation, which in turn make the pyrin dissociate from 14-3-3 and facilitate active pyrin inflammasome formation. These findings are supported by the reports on the dominantly inherited disorder called pyrin-associated autoinflammation with neutrophilic dermatosis (PAAND) $(30,31)$. In patients with PAAND, pyrin S242A or G244L mutation cause a decrease of PKN1/2-dependent pyrin phosphorylation and binding to 14-3-3, triggering the spontaneous pyrin activation. Although this RhoA-dependent pyrin activation model well describes the physiological function of pyrin as a surveillance protein against bacterial toxins without directly recognizing the pathogen-associated molecular patterns, it is difficult to explain the pathological significance of this model since FMF-associated mutations are clustered in PRY/SPRY domain of pyrin. Considering that RhoA-dependent pyrin activation is observed in murine macrophage whose pyrin lacks PRY/SPRY domain (32) and that there are differences in clinical symptoms between FMF and PAAND, it is plausible that PRY/SPRY domain of human pyrin has a function for inflammasome formation in a certain manner other than the RhoA-dependent pathway. As expected, we have discovered the new PRY/SPRY domainbinding regulator, $\beta 2 \mathrm{MG}$, for pyrin inflammasome formation in this study.

B2MG is ubiquitously expressed mainly as a component of MHC class I or CD1 molecules, contributing to antigen presentation. On the other hand, it has been reported that in neutrophil two-thirds of $\beta 2 M G$ molecules are localized in granules, including gelatinase granules and specific granules (33). The function and binding proteins of $\beta 2 M G$ contained in granules of neutrophil had not been known before we identified $\beta 2 M G$ as a pyrin-binding protein in this study. Under normal circumstances, intragranular $\beta 2 \mathrm{MG}$ and cytoplasmic pyrin never interact because of their different localizations. This raises the question of where and how pyrin interacts with $32 \mathrm{MG}$ in the cell. When neutrophils phagocytose foreign bodies resistant to phagosomal digestion such as MSU crystals, phagosomes containing them fuse with granules, eventually rupture, and are unable to digest its contents $(34,35)$. This event is called 
"phagosomal destabilization" and results in the cytosolic distribution of intragranular proteins. Although the phagosome destabilization has been argued mainly for the activation of NLRP3 inflammasome so far, it can also cause the pyrin inflammasome activation as these two inflammasomes can be activated by the same stimulation $(22,36,37)$. Thus, we suspect that the pyrin- $\beta 2 M G$ interaction can be occurred by this phagosomal destabilization before pyrin inflammasome formation. Consistent with this idea, $\beta 2 M G$ was not co-localized with pyrin in human neutrophils without stimulation while their co-localization was observed in neutrophils stimulated with LPS and MSU in the speck-like structures. Pyrin can be considered to function as a cytosolic sensor for phagosomal destabilization by detecting $32 \mathrm{MG}$.

FMF is, as the name suggests, prevalent in Mediterranean descendants and the selective advantage for the MEFV mutation carrier is speculated (38). A recent study showed that FMF mutant pyrin interacts less firmly with a virulence factor YopM of Yersinia pestis, which is an intracellular parasite, than the wild-type pyrin, thereby attenuating YopM-induced interleukin-1 $\beta$ suppression (39). Thus, FMF mutations positively selected in Mediterranean populations increase resistance to $Y$. pestis. Considering other intracellular parasites such as Mycobacterium tuberculosis and Listeria monocytogenes are known to tolerate or escape from the phagolysosomal digestion of macrophages (40), not only crystal structures like MSU but also such pathogenic microorganisms can cause the phagosomal destabilization in neutrophils. Based on our hypothesis, the selective advantage for the MEFV mutation carrier can partly rely on the expected mechanism that $\beta 2 M G$ can be released into the cytoplasm by the trigger due to infection and that following pyrin inflammasome activation may have a pivotal role in the control of infection.

PSTPIP1 harboring PAPA syndrome-associated mutations (A230T and E250Q) presents a reduced affinity for PEST (rich in proline [P], glutamic acid [E], serine [S], and threonine [T])-type protein tyrosine phosphatase (PTP-PEST), resulting in increased phosphorylation of PSTPIP1. Subsequently, the hyperphosphorylated PSTPIP1 strongly binds to pyrin, leading to excessive formation of pyrin inflammasome (26). Although the importance of PSTPIP1 in pyrin inflammasome formation had been elucidated, it was unknown what causes pyrin-PSTPIP1 interaction other than the PAPA syndromeassociated PSTPIP1 mutations. Our findings suggest that the $\beta 2 \mathrm{MG}$ binding to pyrin promotes pyrinPSTPIP1 interaction, which leads to pyrin inflammasome formation. It indicates the possibility that there exists a RhoA-independent pyrin activation pathway.

The present study also suggests the important role of interaction between pyrin and caspase-1 p20 subunit for the control of pyrin inflammasome activation. There are conflicting reports on pyrin-caspase-1 interaction. One study showed all full-length and p20, p10 subunits of caspase- 1 interacted with the pyrin PRY/SPRY domain and that the FMF-associated mutation of pyrin decreased its affinity for caspase-1 (18). On the contrary, another paper reported that the interaction of caspase-1 with the pyrin PRY/SPRY domain with the M694V mutation is equally strong as the one observed with the wild-type, suggesting that the mutation does not affect the binding of pyrin to caspase-1 (20). In our study, the caspase-1 p20 subunit strongly bound to the pyrin PRY/SPRY domain, inhibiting pyrin- $\beta 2 M G$ interaction, and M694V mutation modified this antagonism toward the interaction with $\beta 2 M G$. Thus, we consider that the FMF- 
associated pyrin mutation can cause stronger inflammasome activation via its decreased affinity for caspase-1 and increased interaction with $\beta 2 \mathrm{MG}$.

Based on our findings, here we propose a novel hypothesis for FMF pathogenesis (Fig. 9). B2MG functions as a pyrin ligand inducing pyrin inflammasome formation and subsequent production of active caspase- 1 p20 and p10 subunits, followed by blocking of the $32 M G$-pyrin interaction by p20 in a negative feedback manner. Pyrin M694V mutation disrupts the negative feedback by caspase-1 p20 subunit, resulting in the promotion of pyrin inflammasome formation and the vicious cycle of inflammation.

\section{Conclusion}

We identified the novel pyrin-binding protein, $\beta 2 M G$ and found that pyrin- $\beta 2 M G$ interaction recruits the pyrin inflammasome components, PSTPIP1 and ASC. Although further studies are needed to confirm how $\beta 2 M G$ meets pyrin in the cytoplasm of neutrophils, inhibition of the pyrin- $\beta 2 M G$ interaction can be a promising candidate as a target for FMF treatment.

\section{List Of Abbreviations}

FMF; familial Mediterranean fever, TRIM; tripartite motif-containing, ASC; apoptosis-associated speck-like protein containing caspase recruitment domain, IL-1 $\beta$; interleukin-1 $\beta$, PSTPIP1; proline-serine-threonine phosphatase interacting protein 1, PAPA; pyogenic arthritis, pyoderma gangrenosum and acne, $\beta 2 \mathrm{MG}$; $\beta 2$ microglobulin, FL-pyrin; full-length human pyrin, C-pyrin; C-terminal region of human pyrin, Ab; antibody, MSU; monosodium urate, HEK; human embryonic kidney, MHC; major histocompatibility complex, PAAND; pyrin-associated autoinflammation with neutrophilic dermatosis.

\section{Declarations}

\section{Ethics approval and consent to participate}

The study protocol is approved by the ethics committee of Yokohama City University Hospital (B100701027, B191000004), and informed consent was obtained from the healthy controls. The study was conducted based on the Declaration of Helsinki.

\section{Consent for publication}

Not applicable.

\section{Availability of data and materials}

The datasets analyzed during the current study are available from the corresponding author on reasonable request. 
The authors declare no competing interests.

\section{Funding}

This work was supported by JSPS KAKENHI Grant Numbers JP19K08914 (RY), the Japan Rheumatism Foundation (RY), and SENSHIN Medical Research Foundation (RY).

\section{Authors' contributions}

SS performed experiments with assistance from RY. SS and RY conceived the project, designed the experiments, analyzed the results, prepared figures, and devised the manuscript. YK and HN coordinated and critically discussed research work. All the above-listed authors edited the manuscript.

\section{Acknowledgements}

We thank all the colleagues in our laboratories for helpful cooperation, suggestions and meaningful discussions of this project. We also thank Mr. Tom Kiper for his review of this manuscript.

\section{References}

1. Ben-Chetrit E, Levy M. Familial Mediterranean fever. Lancet. 1998;351:659-64.

2. 1997. A candidate gene for familial Mediterranean fever. Nature genetics 17: 25-31.

3. 1997. Ancient missense mutations in a new member of the RoRet gene family are likely to cause familial Mediterranean fever. The International FMF Consortium. Cel/ 90: 797-807.

4. Mansfield E, Chae JJ, Komarow HD, Brotz TM, Frucht DM, Aksentijevich I, Kastner DL. The familial Mediterranean fever protein, pyrin, associates with microtubules and colocalizes with actin filaments. Blood. 2001;98:851-9.

5. Gack MU, Shin YC, Joo CH, Urano T, Liang C, Sun L, Takeuchi O, Akira S, Chen Z, Inoue S, Jung JU. TRIM25 RING-finger E3 ubiquitin ligase is essential for RIG-I-mediated antiviral activity. Nature. 2007;446:916-20.

6. Yoshimi R, Chang TH, Wang H, Atsumi T, Morse HC 3rd, and Ozato K. Gene disruption study reveals a nonredundant role for TRIM21/Ro52 in NF-kappaB-dependent cytokine expression in fibroblasts. J Immunol. 2009;182:7527-38.

7. Shi M, Deng W, Bi E, Mao K, Ji Y, Lin G, Wu X, Tao Z, Li Z, Cai X, Sun S, Xiang C, Sun B. TRIM30 alpha negatively regulates TLR-mediated NF-kappa $B$ activation by targeting TAB2 and TAB3 for degradation. Nature immunology. 2008;9:369-77.

8. Hatakeyama S. TRIM Family Proteins: Roles in Autophagy, Immunity, and Carcinogenesis. Trends Biochem Sci. 2017;42:297-311.

9. Chang TH, Yoshimi R, Ozato K. Tripartite Motif (TRIM) 12c, a Mouse Homolog of TRIM5, Is a Ubiquitin Ligase That Stimulates Type I IFN and NF-kappaB Pathways along with TNFR-Associated Factor 6. J Immunol. 2015;195:5367-79. 
10. Ozato $\mathrm{K}$, Shin DM, Chang TH, Morse HC 3rd. TRIM family proteins and their emerging roles in innate immunity. Nat Rev Immunol. 2008;8:849-60.

11. Richards N, Schaner P, Diaz A, Stuckey J, Shelden E, Wadhwa A, Gumucio DL. Interaction between pyrin and the apoptotic speck protein (ASC) modulates ASC-induced apoptosis. J Biol Chem. 2001;276:39320-9.

12. Martinon F, Burns K, Tschopp J. The inflammasome: a molecular platform triggering activation of inflammatory caspases and processing of prolL-beta. Molecular cell. 2002;10:417-26.

13. Yu JW, Wu J, Zhang Z, Datta P, Ibrahimi I, Taniguchi S, Sagara J, Fernandes-Alnemri T, Alnemri ES. Cryopyrin and pyrin activate caspase-1, but not NF-kappaB, via ASC oligomerization. Cell death differentiation. 2006;13:236-49.

14. Yu JW, Fernandes-Alnemri T, Datta P, Wu J, Juliana C, Solorzano L, McCormick M, Zhang Z, Alnemri ES. Pyrin activates the ASC pyroptosome in response to engagement by autoinflammatory PSTPIP1 mutants. Molecular cell. 2007;28:214-27.

15. Wise CA, Gillum JD, Seidman CE, Lindor NM, Veile R, Bashiardes S, Lovett M. Mutations in CD2BP1 disrupt binding to PTP PEST and are responsible for PAPA syndrome, an autoinflammatory disorder. Human molecular genetics. 2002;11:961-9.

16. Tunca M, Akar S, Onen F, Ozdogan H, Kasapcopur O, Yalcinkaya F, Tutar E, Ozen S, Topaloglu R, Yilmaz E, Arici M, Bakkaloglu A, Besbas N, Akpolat T, Dinc A, Erken E. Familial Mediterranean fever (FMF) in Turkey: results of a nationwide multicenter study. Medicine. 2005;84:1-11.

17. Weinert C, Grutter C, Roschitzki-Voser H, Mittl PR, Grutter MG. The crystal structure of human pyrin b30.2 domain: implications for mutations associated with familial Mediterranean fever. Journal of molecular biology. 2009;394:226-36.

18. Chae JJ, Wood G, Masters SL, Richard K, Park G, Smith BJ, Kastner DL. The B30.2 domain of pyrin, the familial Mediterranean fever protein, interacts directly with caspase-1 to modulate IL-1beta production. Proc Natl Acad Sci USA. 2006;103:9982-7.

19. Balci-Peynircioglu B, Waite AL, Hu C, Richards N, Staubach-Grosse A, Yilmaz E, Gumucio DL. Pyrin, product of the MEFV locus, interacts with the proapoptotic protein, Siva. Journal of cellular physiology. 2008;216:595-602.

20. Papin S, Cuenin S, Agostini L, Martinon F, Werner S, Beer HD, Grutter C, Grutter M, Tschopp J. The SPRY domain of Pyrin, mutated in familial Mediterranean fever patients, interacts with inflammasome components and inhibits prolL-1 beta processing. Cell death differentiation. 2007;14:1457-66.

21. Seegmiller J, Howell R, Malawista S. The inflammatory reaction to sodium urate. JAMA. 1962;180:469-75.

22. Xu H, Yang J, Gao W, Li L, Li P, Zhang L, Gong YN, Peng X, Xi JJ, Chen S, Wang F, Shao F. Innate immune sensing of bacterial modifications of Rho GTPases by the Pyrin inflammasome. Nature. 2014;513:237-41. 
23. Bjerrum OW, Bjerrum OJ, Borregaard N. Beta 2-microglobulin in neutrophils: an intragranular protein. J Immunol. 1987;138:3913-7.

24. Roberge CJ, Grassi J, De Medicis R, Frobert Y, Lussier A, Naccache PH, Poubelle PE. Crystalneutrophil interactions lead to interleukin-1 synthesis. Agents actions. 1991;34:38-41.

25. Shinar Y, Obici L, Aksentijevich I, Bennetts B, Austrup F, Ceccherini I, Costa JM, De Leener A, Gattorno M, Kania U, Kone-Paut I, Lezer S, Livneh A, Moix I, Nishikomori R, Ozen S, Phylactou L, Risom L, Rowczenio D, Sarkisian T, van Gijn ME, Witsch-Baumgartner M, Morris M, Hoffman HM, Touitou I. Guidelines for the genetic diagnosis of hereditary recurrent fevers. Ann Rheum Dis. 2012;71:1599605.

26. Shoham NG, Centola M, Mansfield E, Hull KM, Wood G, Wise CA, Kastner DL. Pyrin binds the PSTPIP1/CD2BP1 protein, defining familial Mediterranean fever and PAPA syndrome as disorders in the same pathway. Proc Natl Acad Sci USA. 2003;100:13501-6.

27. Schnappauf O, Chae JJ, Kastner DL, Aksentijevich I. The Pyrin Inflammasome in Health and Disease. Frontiers in immunology. 2019;10:1745.

28. Shinar Y, Livneh A, Langevitz P, Zaks N, Aksentijevich I, Koziol DE, Kastner DL, Pras M, Pras E. Genotype-phenotype assessment of common genotypes among patients with familial Mediterranean fever. J Rhuematol. 2000;27:1703-7.

29. Park YH, Wood G, Kastner DL, Chae JJ. Pyrin inflammasome activation and RhoA signaling in the autoinflammatory diseases FMF and HIDS. Nature immunology. 2016;17:914-21.

30. Masters SL, Lagou V, Jeru I, Baker PJ, Van Eyck L, Parry DA, Lawless D, De Nardo D, Garcia-Perez JE, Dagley LF, Holley CL, Dooley J, Moghaddas F, Pasciuto E, Jeandel PY, Sciot R, Lyras D, Webb Al, Nicholson SE, De Somer L, van Nieuwenhove E, Ruuth-Praz J, Copin B, Cochet E, Medlej-Hashim M, Megarbane A, Schroder K, Savic S, Goris A, Amselem S, Wouters C, Liston A. Familial autoinflammation with neutrophilic dermatosis reveals a regulatory mechanism of pyrin activation. Science translational medicine. 2016;8:332ra345.

31. Moghaddas F, Llamas R, De Nardo D, Martinez-Banaclocha H, Martinez-Garcia JJ, Mesa-Del-Castillo P, Baker PJ, Gargallo V, Mensa-Vilaro A, Canna S, Wicks IP, Pelegrin P, Arostegui JI, Masters SL. 2017. A novel Pyrin-Associated Autoinflammation with Neutrophilic Dermatosis mutation further defines 14-3-3 binding of pyrin and distinction to Familial Mediterranean Fever. Annals of the rheumatic diseases 76: 2085-2094.

32. Chae JJ, Centola M, Aksentijevich I, Dutra A, Tran M, Wood G, Nagaraju K, Kingma DW, Liu PP, Kastner DL. Isolation, genomic organization, and expression analysis of the mouse and rat homologs of MEFV, the gene for familial mediterranean fever. Mammalian genome: official journal of the International Mammalian Genome Society. 2000;11:428-35.

33. Borregaard N, Sorensen OE, Theilgaard-Monch K. Neutrophil granules: a library of innate immunity proteins. Trends Immunol. 2007;28:340-5.

34. Hornung V, Bauernfeind F, Halle A, Samstad EO, Kono H, Rock KL, Fitzgerald KA, Latz E. Silica crystals and aluminum salts activate the NALP3 inflammasome through phagosomal destabilization. Nature 
immunology. 2008;9:847-56.

35. Martinon F, Petrilli V, Mayor A, Tardivel A, Tschopp J. Gout-associated uric acid crystals activate the NALP3 inflammasome. Nature. 2006;440:237-41.

36. Gavrilin MA, Abdelaziz DH, Mostafa M, Abdulrahman BA, Grandhi J, Akhter A, Abu Khweek A, Aubert $D F$, Valvano MA, Wewers MD, Amer AO. Activation of the pyrin inflammasome by intracellular Burkholderia cenocepacia. J Immunol. 2012;188:3469-77.

37. Rosales-Reyes R, Aubert DF, Tolman JS, Amer AO, Valvano MA. Burkholderia cenocepacia type VI secretion system mediates escape of type II secreted proteins into the cytoplasm of infected macrophages. PloS one. 2012;7:e41726.

38. Aksentijevich I, Torosyan Y, Samuels J, Centola M, Pras E, Chae JJ, Oddoux C, Wood G, Azzaro MP, Palumbo G, Giustolisi R, Pras M, Ostrer H, Kastner DL. Mutation and haplotype studies of familial Mediterranean fever reveal new ancestral relationships and evidence for a high carrier frequency with reduced penetrance in the Ashkenazi Jewish population. Am J Hum Genet. 1999;64:949-62.

39. Park YH, Remmers EF, Lee W, Ombrello AK, Chung LK, Shilei Z, Stone DL, Ivanov MI, Loeven NA, Barron KS, Hoffmann P, Nehrebecky M, Akkaya-Ulum YZ, Sag E, Balci-Peynircioglu B, Aksentijevich I, Gul A, Rotimi CN, Chen H, Bliska JB, Ozen S, Kastner DL, Shriner D, Chae JJ. Ancient familial Mediterranean fever mutations in human pyrin and resistance to Yersinia pestis. Nature immunology. 2020;21:857-67.

40. Portnoy DA, Auerbuch V, Glomski IJ. The cell biology of Listeria monocytogenes infection: the intersection of bacterial pathogenesis and cell-mediated immunity. J Cell Biol. 2002;158:409-14.

\section{Figures}

\section{Figure 1}

full-length pyrin

(FL-pyrin; 1-781)
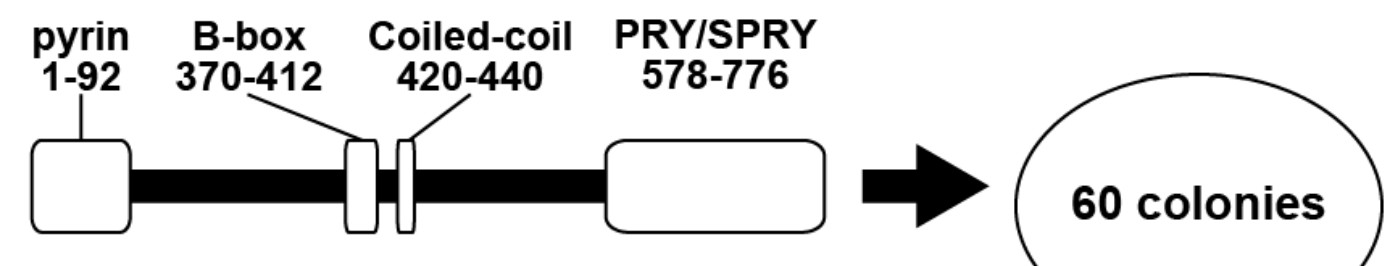

C-terminal pyrin

(C-pyrin; 598-781)
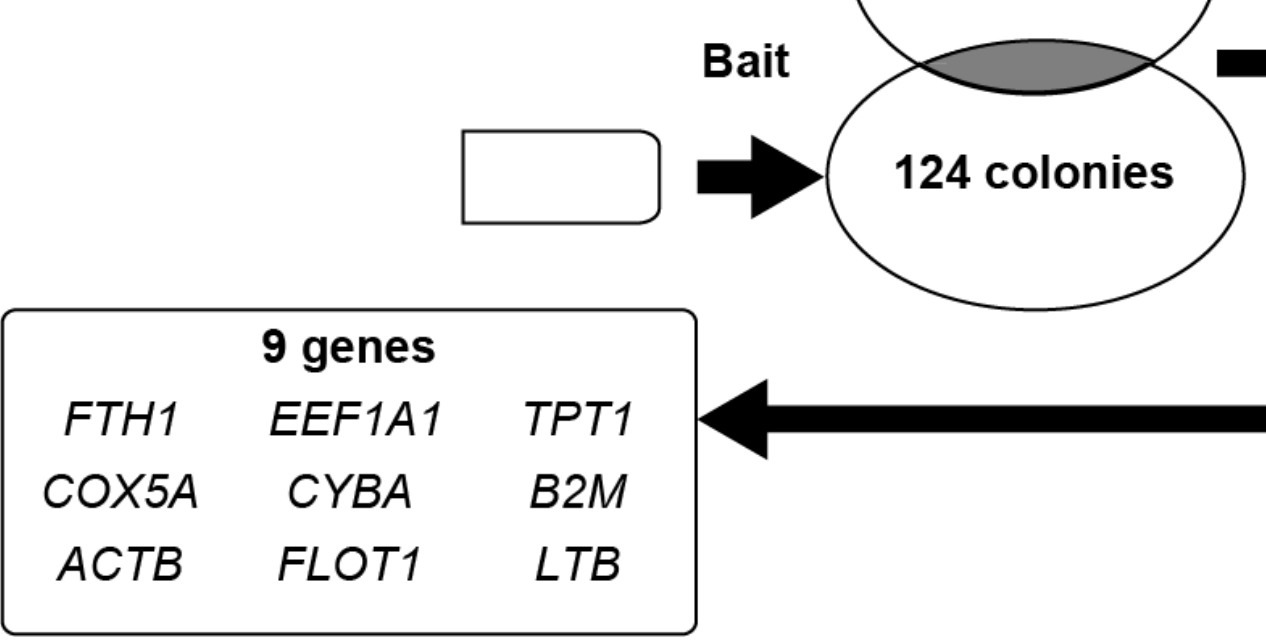
Figure 1

Yeast two-hybrid screening for pyrin-binding proteins. The yeast AH109 was transformed with Full-length pyrin (FL-pyrin) or C-terminal pyrin (C-pyrin) inserted in pGBKT7 vector as baits. As prey, human leukocyte cDNAs were inserted in pACT2 and transfected to yeast Y187 strain. After mating, FL-pyrin and C-pyrin baits yielded 60 and 124 positive colonies, respectively. Nine common genes were identified from both FL- and C-pyrin baits.

Figure 2
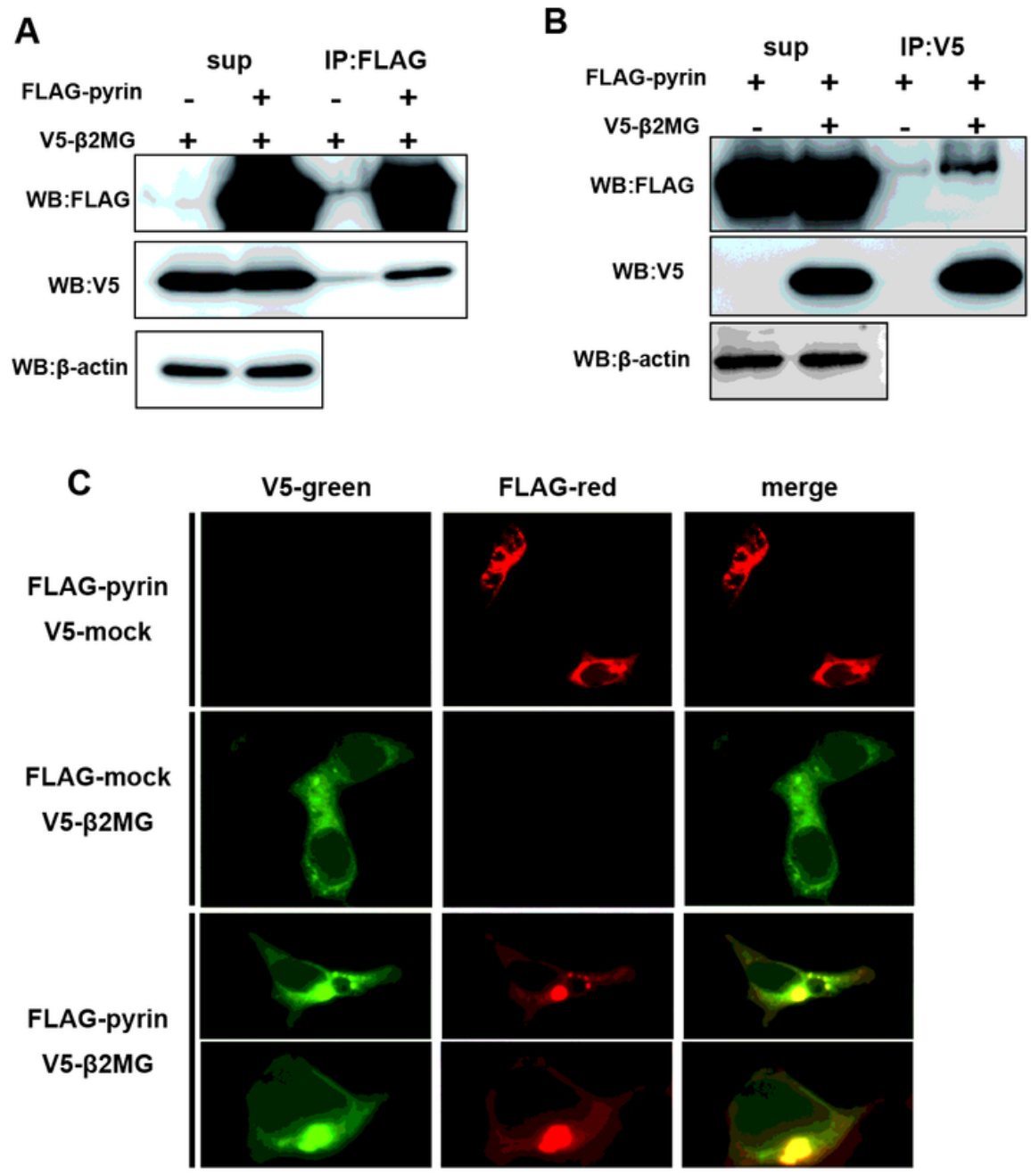

Figure 2 
Pyrin interacts with $\beta 2 \mathrm{MG}$ in mammalian cells. A, B, Lenti-X 293T cells expressing FLAG-tagged pyrin (FLAG-pyrin) and V5-tagged $\beta 2 M G$ (V5- $32 \mathrm{MG}$ ) were lysed and immunoprecipitated by FLAG Abconjugated agarose beads or $\mathrm{V} 5 \mathrm{Ab}$-conjugated magnet beads, and then subjected to immunoblot analysis. V5- $\beta 2 M G$ is co-immunoprecipitated with FLAG-pyrin (A) and vice versa (B). C, HEK293 cells expressing FLAG-pyrin (red) and V5- $\beta 2 M G$ (green) were subjected to immunofluorescence staining. Pyrin and $\beta 2 M G$ shows co-localization in the cytoplasm.

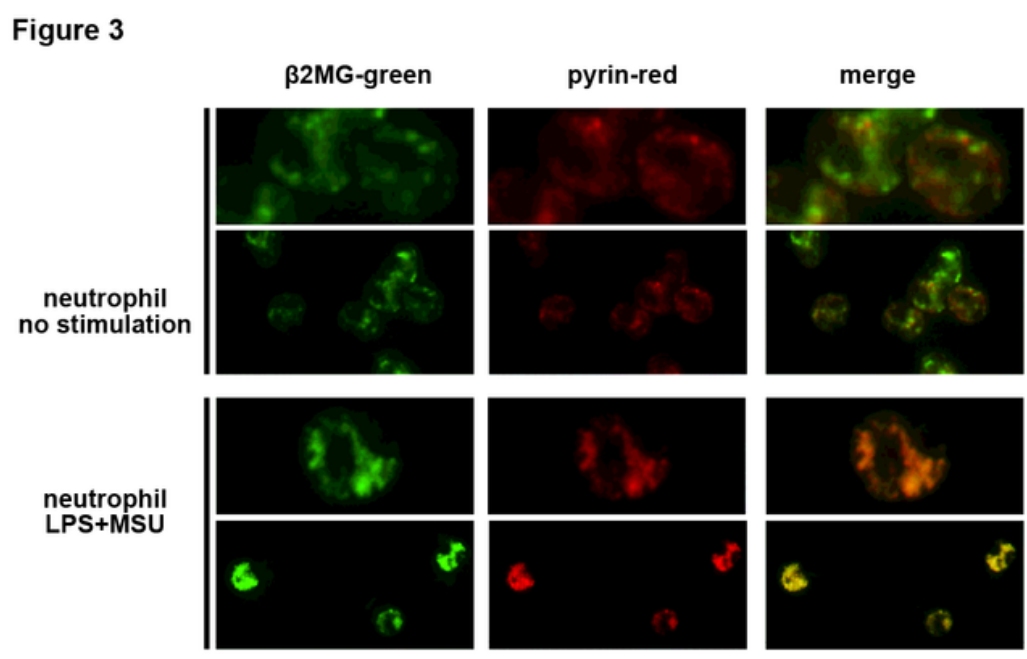

\section{Figure 3}

Pyrin is co-localized with $\beta 2 M G$ in MSU-stimulated neutrophils. Human neutrophils stimulated with LPS and MSU crystals were subjected to immunofluorescence staining analyses. Pyrin (red) and $\beta 2 M G$ (green) are co-localized in cytoplasm, with forming speck, in the stimulated neutrophils while they are separately localized in cytoplasm and perinuclear granules, respectively, in the unstimulated neutrophils. 
Figure 4

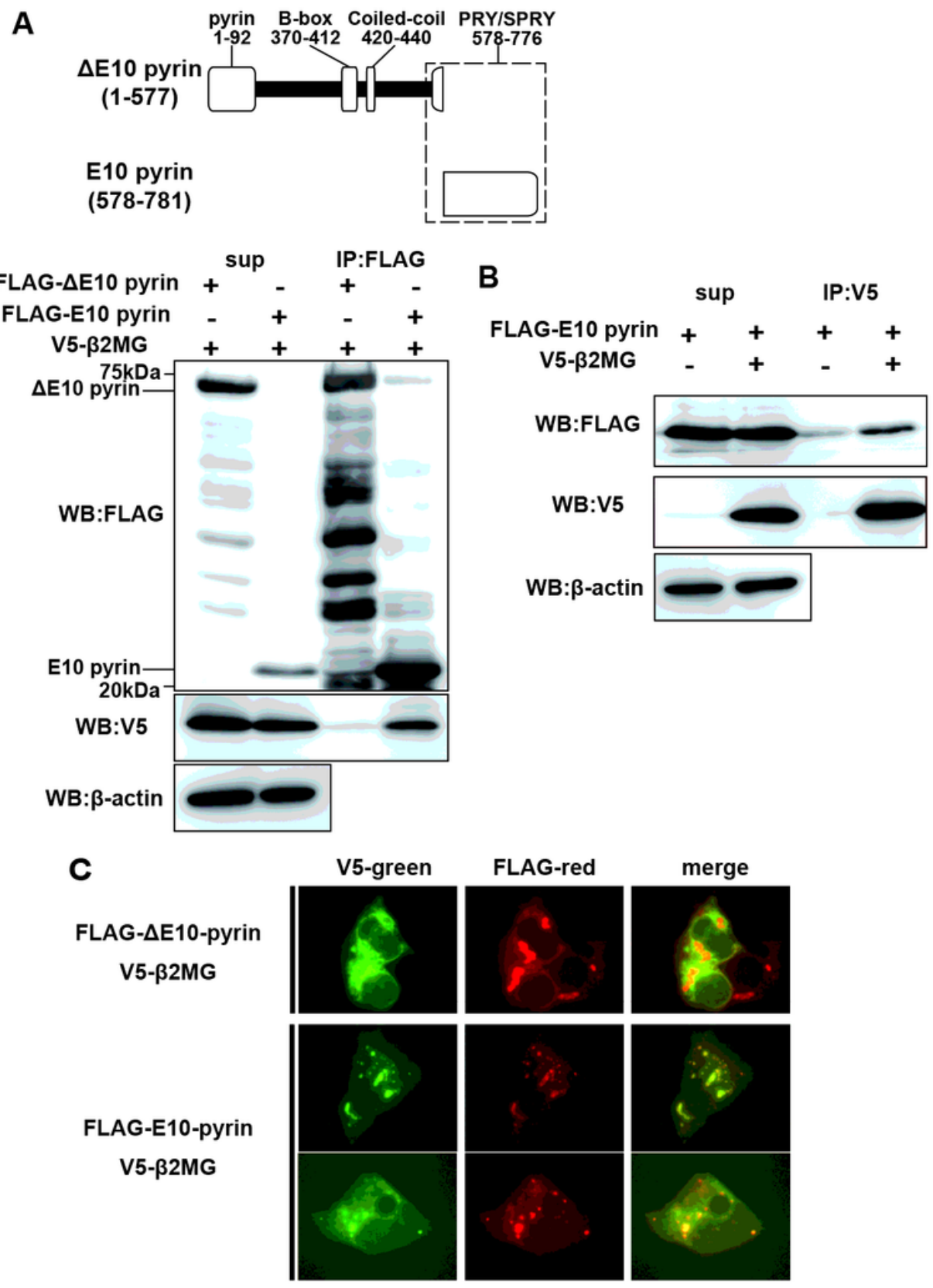

Figure 4

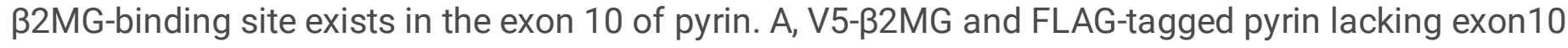
(FLAG- 10 pyrin) or FLAG-tagged pyrin exon10 (FLAG-E10 pyrin) were expressed in Lenti-X 293T cells. The cells were lysed with lysis buffer and immunoprecipitated by FLAG Ab-conjugated agarose beads. Co-immunoprecipitation analysis reveals V5-B2MG binds to the pyrin exon 10 region. B, Lenti-X 293T cells expressing FLAG-E10 pyrin and V5- $32 M G$ were subjected to immunoprecipitation analysis using V5 Abconjugated magnetic beads. The co-immunoprecipitation analysis confirms the interaction of the pyrin exon10 region and $\beta 2 M G$. C, HEK293 cells expressing FLAG- $\triangle E 10$ or FLAG-E10 pyrin and V5- $\beta 2 M G$ were subjected to immunofluorescence staining analysis. V5-B2MG (green) was colocalized with FLAG-E10 
pyrin (red) in the speck-like structures in the perinuclear position in more than $90 \%$ of the overexpressed cells while no co-localization of V5- $\beta 2 M G$ with FLAG- $\Delta$ E10 (red) was observed.

\section{Figure 5}

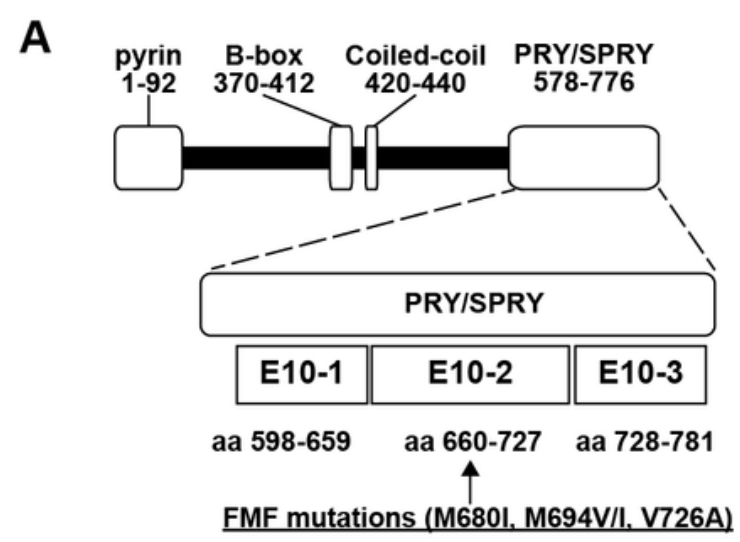

B

sup

IP:FLAG

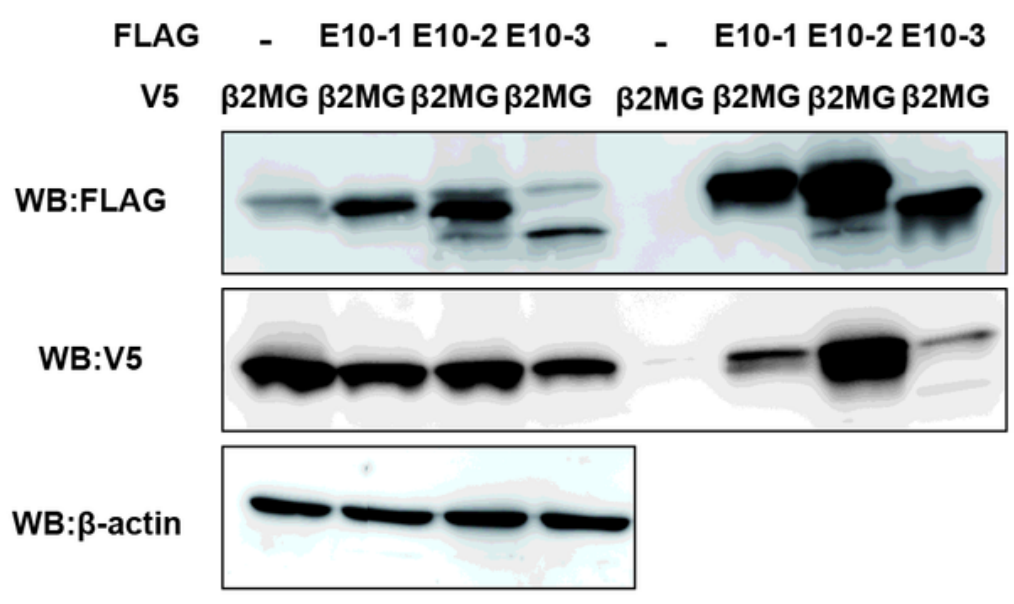

\section{Figure 5}

$\beta 2 M G$ binds to the FMF mutation site. A, Major MEFV mutations causing familial Mediterranean fever, M680I, M694I/V, and V726A, are concentrated in the middle portion of exon10 region (E10-2). B, Lenti-X 293T cells expressing V5-32MG and FLAG-tagged pyrin 10-1 (E10-1), 10-2 (E10-2), or 10-3 region (E10-3) 
were lysed with lysis buffer, and subjected to co-immunoprecipitation analysis using FLAG Ab-conjugated agarose beads. Co-immunoprecipitation analysis reveales that $\beta 2 \mathrm{MG}$ binds to E10-2, which includes the hotspot of FMF-associated mutations.

Figure 6

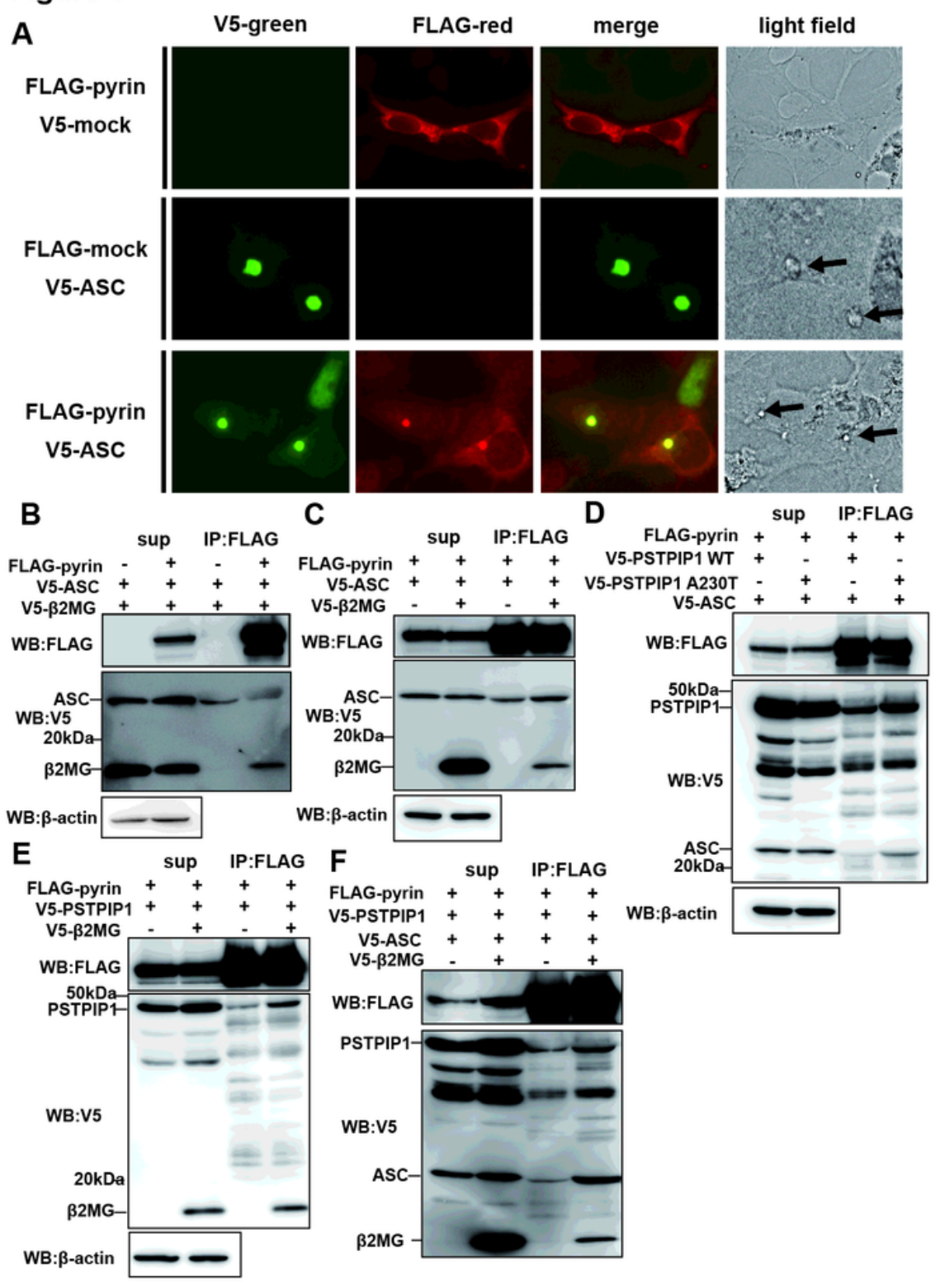

\section{Figure 6}

B2MG promotes PSTPIP1-mediated pyrin inflammasome formation. A, HEK 293 cells expressing FLAGpyrin and V5-tagged ASC were subjected to immunofluorescence staining analysis, which revealed colocalization of pyrin (red) and ASC (green) with the speck formation in more than $90 \%$ of the 
overexpressed cells. B, Lenti-X 293T cells expressing V5-ASC and V5- 32 MG with or without FLAG-pyrin were lysed with lysis buffer and subjected to co-immunoprecipitation analysis using FLAG Ab-conjugated agarose beads. Co-immunoprecipitation analysis reveals that pyrin does not associate with ASC. C, LentiX 293T cells expressing FLAG-pyrin and V5-ASC with or without V5- $\beta 2 M G$ were lysed with lysis buffer and subjected to co-immunoprecipitation analysis using FLAG Ab-conjugated agarose beads. Coimmunoprecipitation analysis reveals that pyrin- $\beta 2 M G$ binding does not affect pyrin-ASC interaction. D, Lenti-X 293T cells expressing FLAG-pyrin and V5-ASC with V5-tagged wild-type or A230T PSTPIP1 were lysed with lysis buffer and subjected to co-immunoprecipitation analysis by FLAG Ab-conjugated agarose beads. A230T PSIPTP1 binds to pyrin more strongly than the wild-type, leading to the enhanced recruitment of ASC. E, Lenti-X 293T cells expressing FLAG-pyrin and V5-PSTPIP1 with or without V5$\beta 2 M G$ were lysed with lysis buffer and subjected to co-immunoprecipitation analysis using FLAG Abconjugated agarose beads. Co-immunoprecipitation analysis reveals that $\beta 2 \mathrm{MG}$ promotes pyrin-PSTPIP1 interaction. F. Lenti-X 293T cells expressing FLAG-pyrin, V5-PSTPIP1, and V5-ASC with or without V5$\beta 2 M G$ were subjected to co-immunoprecipitation analysis. $\beta 2 M G$ promotes the pyrin-ASC interaction in the presence of PSTPIP1. 
Figure 7
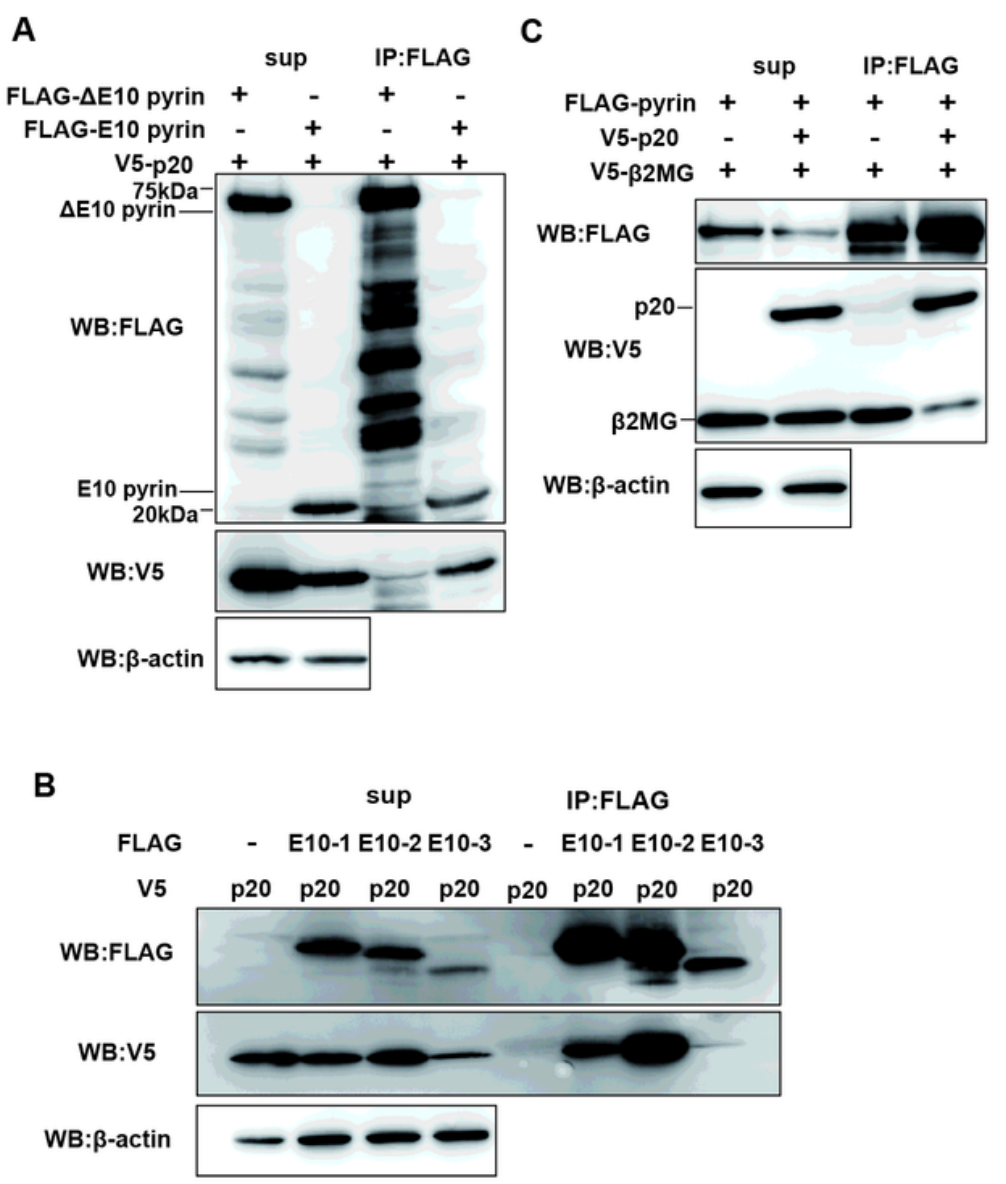

\section{Figure 7}

Caspase-1 p20 subunit interacts with pyrin in a manner that competes with $\beta 2 \mathrm{MG}$. A, FLAG- $\Delta 10$ pyrin and FLAG-E10 pyrin were expressed with V5-tagged caspase-1 p20 subunit (V5-p20) respectively in Lenti-X 293T cells. They were lysed with lysis buffer and subjected to co-immunoprecipitation analysis using FLAG Ab conjugated agarose. V5-p20 binds to pyrin exon10 region. B, Lenti-X 293T cells expressing each pyrin E10-1, E10-2 and E10-3 region with V5-p20 were lysed with lysis buffer. Co-immunoprecipitation analysis using FLAG Ab conjugaed agarose reveals caspase-1 p20 binds to the pyrin E10-2 region, the same as $\beta 2 M G$. C, Lenti-X 293T cells expressing FLAG-pyrin and V5- $\beta 2 M G$ with or without V5-p20 were lysed with lysis buffer and subjected to co-immunoprecipitation analysis using FLAG Ab conjugated 
agarose. Co-immunoprecipitation analysis reveals caspase-1 p20 subunit binds to pyrin competitively with $\beta 2 M G$ and inhibits pyrin- $\beta 2 M G$ binding.

Figure 8
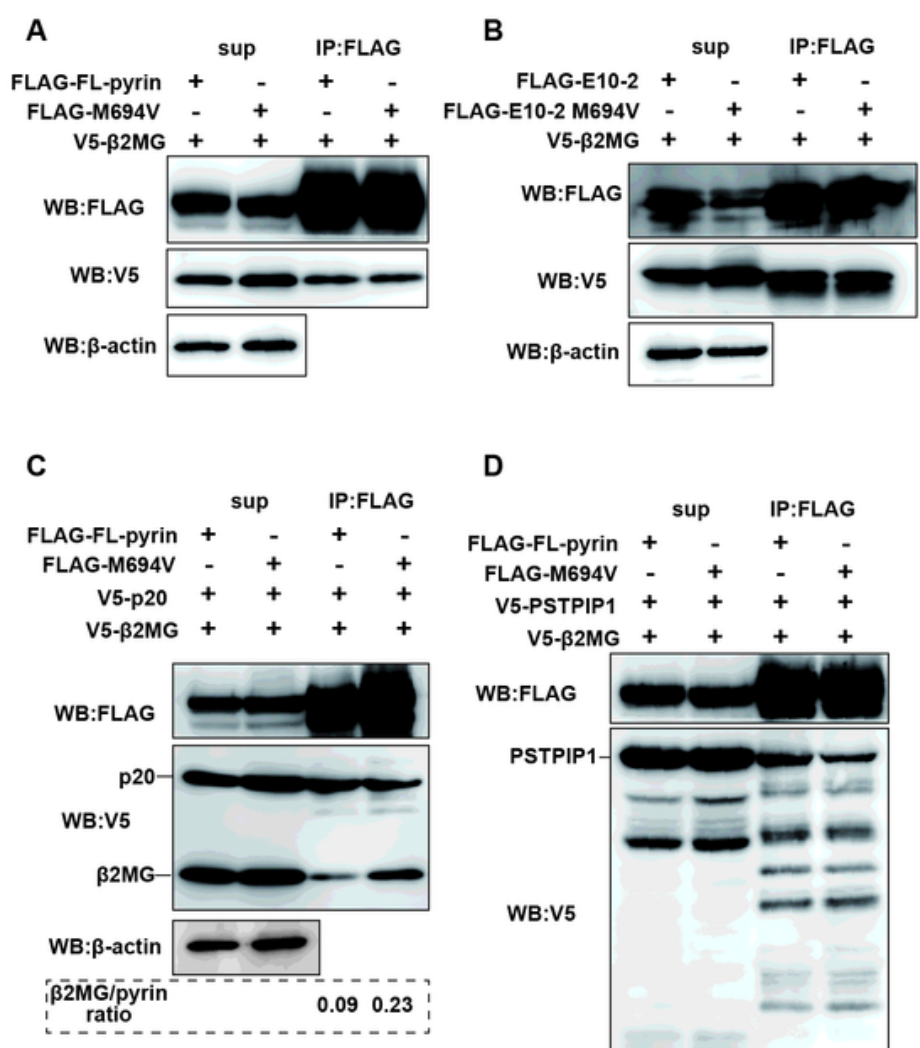

D

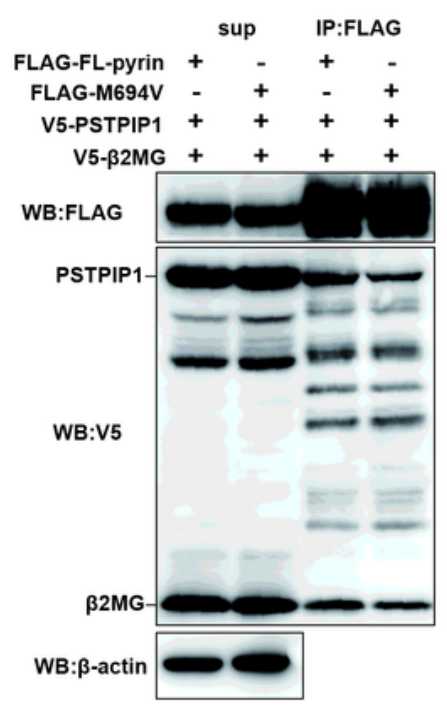

Figure 8

M694V mutation of pyrin weakens the competitive binding of p20 and promotes the recruitment of PSTPIP1 in the presence of $\beta 2 \mathrm{MG}$. A, Lenti-X 293T cells expressing FLAG-tagged full-length (FL) pyrin or M694V pyrin with V5- $32 \mathrm{MG}$ were lysed with lysis buffer and subjected to co-immunoprecipitation 
analysis using FLAG Ab-conjugated agarose. M694V mutation does not affect pyrin- $\beta 2 \mathrm{MG}$ affinity. B, Lenti-X 293T cells expressing FLAG-tagged pyrin deletion mutant E10-2 or M694V-mutated E10-2 with V5B2MG were lysed with lysis buffer and subjected to co-immunoprecipitation analysis by FLAG Abconjugated agarose beads. M694V mutation does not affect the affinity of E10-2 for $\beta 2 M G$. C, Lenti-X 293T cells expressing FLAG-tagged FL- or M694V pyrin and V5- $\beta 2 M G$ with or without V5-p20 were lysed with lysis buffer and subjected to co-immunoprecipitation analysis using FLAG Ab-conjugated agarose. Co-immunoprecipitation analysis reveals that M694V pyrin is prone to bind $32 \mathrm{MG}$ in spite of the presence of p20. D, Lenti-X 293T cells expressing FLAG-tagged FL- or M694V pyrin with V5-PSTPIP1 and V5- 32 MG were lysed with lysis buffer and subjected to co-immunoprecipitation analysis using FLAG Ab-conjugated agarose beads. Co-immunoprecipitation analysis shows no difference in the recruitment of PSTPIP1 between the wild type and M694V pyrin.

\section{Figure 9}

pyrin(wild type)

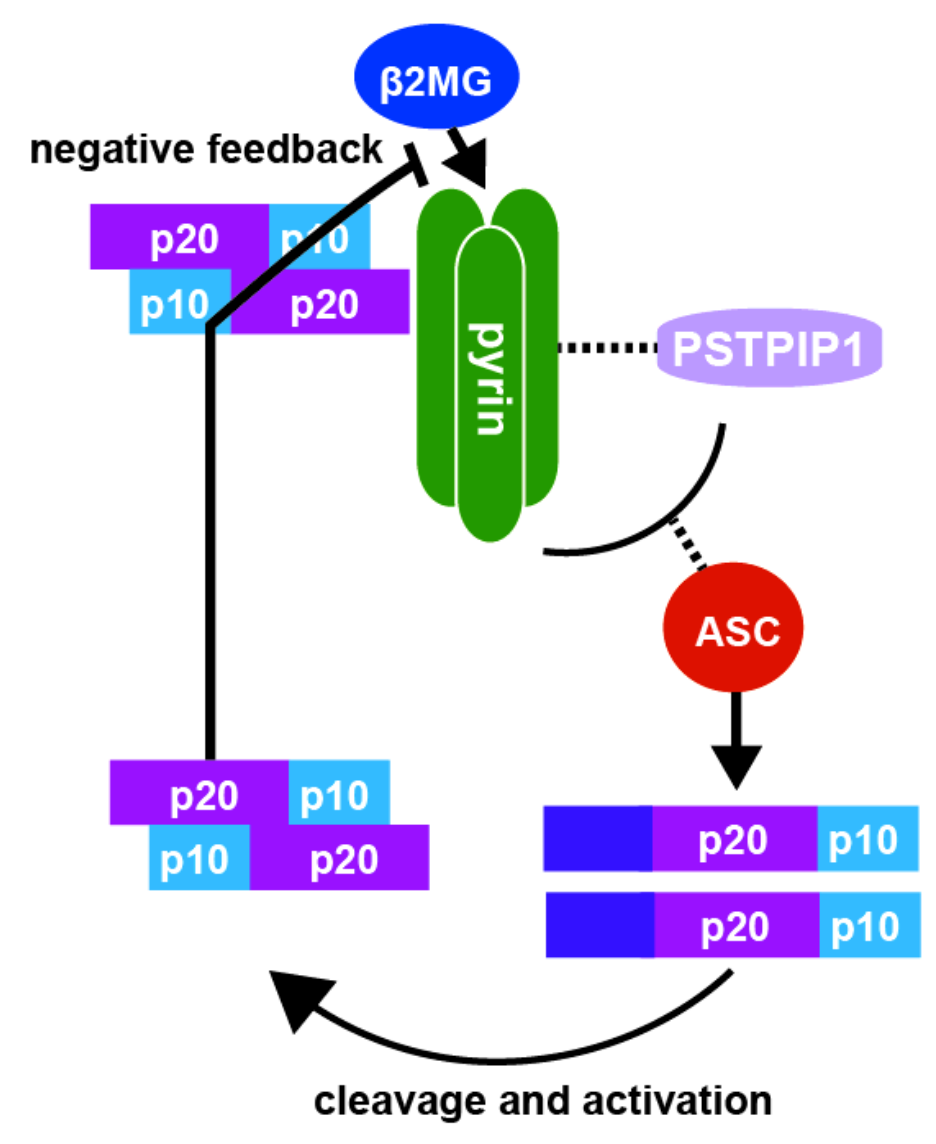

pyrin(M694V)

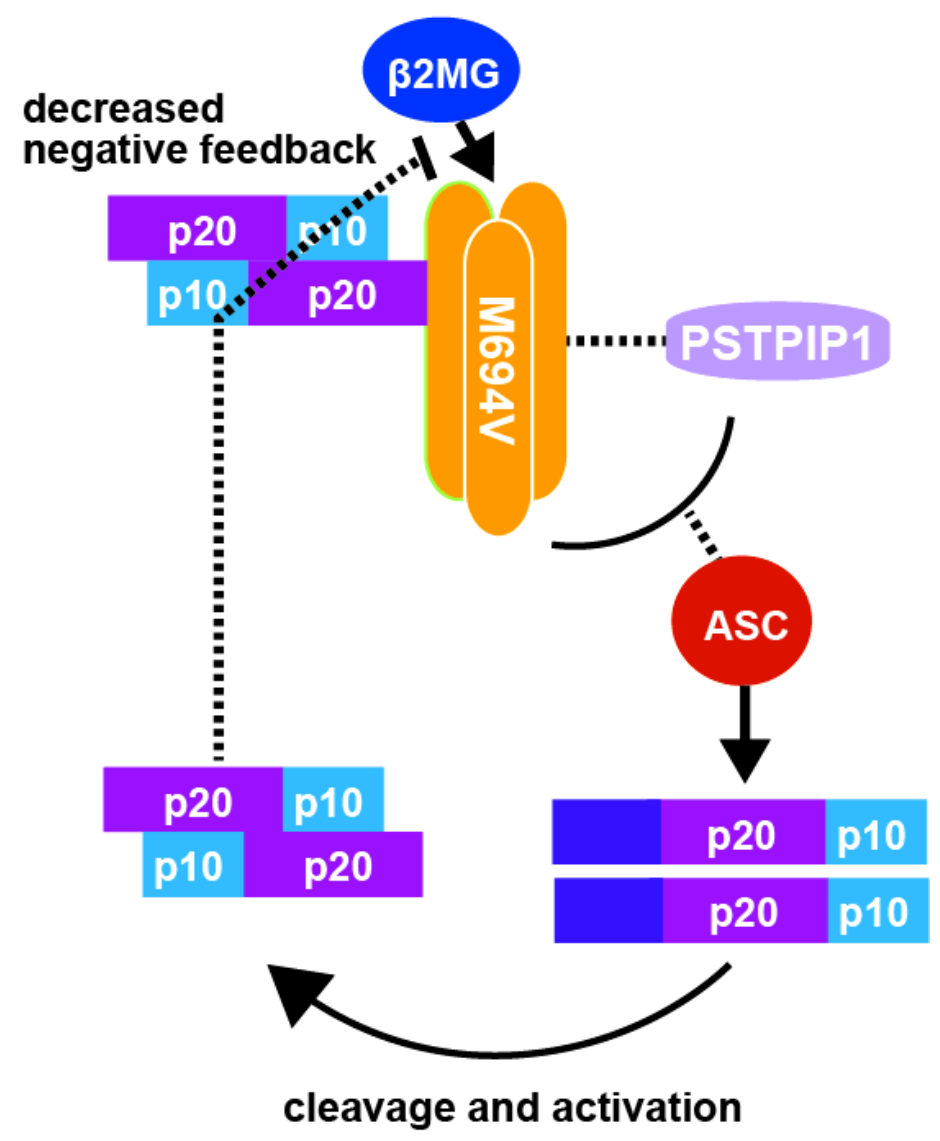

Figure 9

Schematic view of possible mechanisms of pyrin inflammasome regulation and role of MEFV mutation in FMF. The pyrin- $\beta 2 M G$ interaction recruits PSTPIP1 and subsequently ASC, leading to pyrin inflammasome formation. The pyrin inflammasome activates caspase-1, and then the active caspase- 1 subunit 220 suppresses the pyrin- $\beta 2 M G$ interaction in a negative feedback manner. M694V mutation of 
pyrin enhances pyrin inflammasome formation and weakens the negative feedback by p20, resulting in the excessive formation of pyrin inflammasome.

\section{Supplementary Files}

This is a list of supplementary files associated with this preprint. Click to download.

- SupplementalTable1.docx 